\title{
COMMUNAUTÉ DE SOINS
}

Séréna Niel

Médecine \& Hygiène | «Revue internationale de soins palliatifs »

2018/1 Vol. 33 | pages 3 à 4

ISSN 1664-1531

Article disponible en ligne à l'adresse :

https://www.cairn.info/revue-infokara-2018-1-page-3.htm

\section{Pour citer cet article :}

Séréna Niel, "Communauté de soins », Revue internationale de soins palliatifs 2018/1 (Vol. 33), p. 3-4. DOI 10.3917/inka.181.0003

Distribution électronique Cairn.info pour Médecine \& Hygiène.

(C) Médecine \& Hygiène. Tous droits réservés pour tous pays.

La reproduction ou représentation de cet article, notamment par photocopie, n'est autorisée que dans les limites des conditions générales d'utilisation du site ou, le cas échéant, des conditions générales de la licence souscrite par votre établissement. Toute autre reproduction ou représentation, en tout ou partie, sous quelque forme et de quelque manière que ce soit, est interdite sauf accord préalable et écrit de l'éditeur, en dehors des cas prévus par la législation en vigueur en France. Il est précisé que son stockage dans une base de données est également interdit. 


\section{Communauté de soins}

\section{Séréna Niel}

Infirmière Ressource, Maison de Retraite de Petit Saconnex, Genève

Chargée de Cours, Haute École de Santé de Genève HES-SO

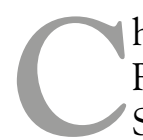

haque nouvelle publication de la Revue internationale francophone de Soins Palliatifs (RISP) est l'occasion de découvrir des pratiques qui, confrontées à la sienne, conduisent à s'interroger sur son quotidien au sein de cette communauté des soins palliatifs.

Dans ce premier numéro de l'année 2018, il est question de la souffrance psychologique, celle des hommes en particulier, un sujet de recherche fort et peu abordé dont la complexité encourage à poursuivre et approfondir l'étude.

La douleur cancéreuse et de son évaluation est aussi abordée dans ce numéro. L'accent est porté sur le rôle du médecin généraliste, pivot des systèmes de santé, dont l'intelligence émotionnelle peut être utilisée comme une ressource thérapeutique.

Il est ensuite question des équipes, multidisciplinaires et interdisciplinaires, dont l'élargissement vers la communauté et sa culture propre vient enrichir la palette de prestations.

Enfin, le monde de la grande vieillesse au sein des établissements spécialisés est dépeint avec l'objectif de garder les yeux et les oreilles grands ouverts.

Et de part et d'autre de ces textes, comme le cadre autour du tableau, c'est le président nouvellement élu de la Fédération Internationale Francophone de Soins Palliatifs, le Professeur Benoît Burucoa, qui nous emmène vers le futur, au-delà de journées au confort inégal.

Ensemble nous avons toutes et tous comme intérêts communs que les soins palliatifs fassent complètement partie des projets de santé de nos sociétés. Cette vision peut aujourd'hui s'opposer au concept initial des soins palliatifs, parfois caractérisés dans une certaine mesure par un clivage entre une phase dite curative et une phase à proprement parler palliative.

Cette dernière conception a conduit une portion non négligeable du public et du monde médical à assimiler les soins palliatifs à des soins terminaux, qualifiés aussi de soins de fin de vie. Les représentations d'arrêt des soins, de retrait thérapeutique, d'abandon du patient, d'il n'y a plus rien à faire, ont constitué et constituent encore des barrières à l'accès aux soins palliatifs. Ceci explique le recours souvent beaucoup trop tardif à ce type de soins. Or, une prise de conscience de plus en plus importante se fait progressivement.

L'objectif est à présent d'intégrer les traitements dirigés contre la maladie aux traitements visant l'amélioration de la qualité de la vie. Il est nécessaire d'adapter leurs contributions respectives en fonction des besoins des patients et de les évaluer avec précision en se référant bien entendu, à la définition de l'OMS déjà publiée en 2002.

De nombreuses études ont montré que les bénéfices des soins palliatifs ne concernent pas uniquement la qualité de la vie et la satisfaction des patients, mais impliquent également une réduction des coûts des soins de santé. De plus, les soins palliatifs seraient même susceptibles de contribuer à un allongement de la durée de la vie. À l'heure actuelle, ce n'est plus tant l'intérêt des soins palliatifs précoces qui est questionné, mais plutôt la manière de les intégrer au mieux en médecine dans le parcours de soins. 
Selon Marie-Françoise Colliere, «Soigner, c'est entretenir la vie en assurant la satisfaction d'un ensemble de besoins indispensables à la vie mais qui sont diversifiés dans leurs manifestations ». [1]

La raison d'être des soins palliatifs est de soulager la souffrance de tout être humain lorsque la

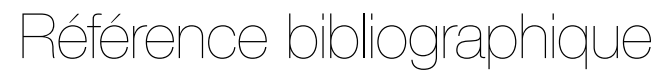

1. Colliere MF. Soigner... Le premier art de la vie. www.elseviermasson.fr/soigner-le-premier-art-de-la-vie-9782294005558.html. perspective de la guérison séloigne et de l'accompagner jusqu'au bout de sa vie avec ses proches.

Un vœu pour ce début de nouvelle année: que les soins palliatifs soient sincèrement intégrés, visibles et universellement reconnus car, appartenir à cette communauté de soins est une fierté! 